\title{
A Semiotic Approach to Critical Reasoning
}

\author{
Janos J. Sarbo ${ }^{1}$ and Jessica H. Yang ${ }^{2}$ \\ ${ }^{1}$ Radboud University Nijmegen, ICIS, NL \\ janos@cs.ru.nl \\ ${ }^{2}$ Henley Business School, Reading, UK \\ j.h.yangahenley.ac.uk
}

\begin{abstract}
Information can be interpreted as in-formation, which refers to the potential of the form for a mediation of meaning. In this paper we focus on reasoning information and consider the question how form involved in reasoning can be used for an analysis of accounting narratives in corporate disclosures. An evaluation of experimental results is included.
\end{abstract}

Keywords: Semiotic, reasoning, rhetoric, argument, accounting narratives.

\section{Introduction}

The Latin word 'informatio' equivalently designates information and bringing-intoform (cf. in-formation). The second refers to the potential of the form for the mediation of meaning (cf. interpreted information). In an extreme case, form and interpretation can be uniquely related. This is parodied by the classical joke of a psychiatric institute in which patients tell stories to each other. As the stories are told frequently they are assigned a number. One of the patients says 'number 42', which gets a big laugh. Another patient cries out 'number 68'. Everybody finds it hilarious. A new patient watching this cannot stop himself and shouts 'number 232'! The entire public bursts into a huge laugh. 'Why are you laughing so much?' the patient asks. His neighbor responds: 'We haven't heard this joke before.'

In everyday language use, form and intended meaning are rarely so closely related. In most cases we are in need of auxiliary words and more refined (formal) expressions for the communication of our thoughts. How does form mediate meaning? Traditional semiotics, which is concerned with interpretation enabled by the sign, does not consider this question. In this paper we suggest that an answer can be given by considering the sign to be an object, and following this line of thought, through introducing a form related, and in this sense, formal model of sign interpretation.

Form involved in information can be used for a derivation of meaning. In the example above, saying a number can be satisfactory as long as the relation between numbers and stories is known by the receiver. If the receiver is not familiar with this relation, the sender may have to use additional information, e.g. syntactic information, and so, a more elaborate form of expression in order to enable the interpretation intended to arise. Besides syntactic information hence a syntactic perspective, many 
other types of perspectives are possible such as a semantical, mathematical, and logical one, that all involve rules of interpretation including those that are related to the form.

If complex thoughts are to be communicated, for instance, an entire text, a sequence of signs is needed. In this case, an important goal is to enable the reader to reveal the line of reasoning by the writer. Reasoning too can be associated with form. In this paper we ask: what are the types of reasoning information, what are the involved forms, and what are the effects of the form on the mediation of reasoning? The answer suggested by this paper is briefly discussed below.

Sign interpretation can be modeled as a process. An analysis from the perspective of reasoning reveals the potential of the events of this process for a representation of the three types of reasoning, deduction, induction, and abduction, as sign aspects. The involved form can be associated with Aristotle's three syllogistic figures as a structure. Through their type of reasoning, the events of the process can be assigned a degree of truthfulness: necessarily true (cf. deduction), expectedly true (cf. induction), and hypothetically true (cf. abduction). By linking the forms of reasoning with their associated claims to truth, the process model can be used for measuring the plausibility and understandability of a sign, e.g., an accounting narrative.

Sign aspects are representations that are in a process of becoming a sign. The development of meaning through sign aspects can be explained by the metaphor of apparent motion perception. In that phenomenon, a series of steady pictures are presented and, although each picture may be meaningful in itself, combined they are interpreted as parameters in the experience of the series of pictures as motion. Snapshots correspond to a sign aspect, the experience of motion to a meaningful interpretation of a sign.

An advantage of the semiotic approach is its potential for a uniform representation for sign interpretation. ${ }^{1}$ This enables syntactic information (e.g. required by a reasoning analysis) and reasoning information to be combined easily. Because of the involvement of the relation between formal and meaningful interpretation, we assume that through analyzing the form, we may draw conclusions about a possible meaning, e.g. from the syllogistic structure of a text about a type of reasoning.

This paper proposes the use of the process-oriented semiotic approach, as an alternative text-focused approach, to interpret corporate disclosures. Corporate disclosures constitute an important channel for organizations to communicate with the public users since it contains rich content of information. Decision-making is built on efficient and effective processing of financial and non-financial (narrative) information [1]. The paper is based on the premise that goal of a critical corporate disclosure should be to reach conclusions by means of reasoned argument.

Research on understandability of accounting narratives in corporate disclosure has gained increasing importance over the years [2,3]. Prior studies can be categorized into two groups measured by content (thematic analysis) [4,5,6,13] and form (syntactical analysis) [7,8,9]. Thematic content analysis is to 'extract and analyze themes inherent within the message' [11] so as to find trends, correlations or attitudes from the text. Syntactical studies adopt readability formulas such as Flesch index,

\footnotetext{
${ }^{1}$ For a number of perspectives (cf. knowledge domains), including those mentioned earlier, this has been illustrated in [10].
} 
Dale-Chall index, and Fog index to measure the probable readability of texts from the perspective of syntactical complexity. This study fills in a gap in the literature on corporate disclosures and communication research in relation to the meaning interpretation of accounting narratives. This approach shows advantages over other traditional ones, in a high degree of focus on the form and process of disclosures rather than on the text itself.

The structure of the paper is the following. First we briefly delve into Peircean semiotics (sect. 2). This is followed by an introduction of a process model of sign interpretation (sect. 3). Finally we offer an explorative study of our model to an analysis of accounting narratives i.e. chairman statements (sect. 4). We close the paper with a summary of the results (sect. 5).

\section{Signs and Interpretation}

Peircean semiotics is founded in Peirce's theory of categories [14]. Contrary to the Aristotelian view that phenomena can be classified in two types, attributes and substances (that are a carrier of attributes), Peirce distinguished phenomena in three categories, firstness, secondness, and thirdness, that have a relational character. An example of phenomena is a letter- $\mathrm{V}$ formed by a pair of fingers. The two fingers, their shape, incidence, etc., as a quality, are a firstness; ad-hoc relations between the qualities, such as the simultaneity involved in the incidence of the two fingers, are a secondness; meaningful interpretations of a relation, such as the interpretation of the incidence relation of the fingers as the symbol of victory, are a thirdness. The categories are ordered according to a relation of involvement, e.g. simultaneity (secondness) is involved in the interpretation of the letter- $\mathrm{V}$ as a symbol of victory.

Beyond the above dependency between the categories, we are interested in the process generating meaningful interpretations (thirdness) from qualities (firstness), through a mediation of relations (secondness). In order to develop a model for this process we need to introduce a few assumptions that are concerned with the questions how qualities may appear, how they may establish relations in an ad-hoc, and a meaningful sense.

In our model below we assume that qualities appear via interactions with the interpreting system (e.g. the human). In an interaction, the qualities of the stimulus (cf. effect) affect the interpreting system occurring in some state. This effect (q1) and state (q2), that both are collections of independent qualities, define the input for information processing (cf. firstness). The goal of interpretation is establishing a relation: why this effect is occurring to this state. To this end, the interpreting system has to sort out the two types of qualities in the input, abstract them from one another in order to determine their interpretation in the light of complementary or context information (cf. secondness), and finally, establish a relation between the input qualities in context, through predication (cf. thirdness). See Fig.1. 


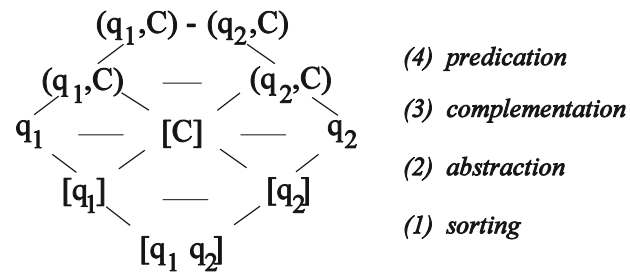

Fig. 1. The process model of cognitive activity. State (q1), effect (q2), context (C). Horizontal lines designate relations. The types of interpretation events are displayed on the right-hand side, in italics. Square brackets indicate that an entity is not yet interpreted, usual bracket symbols and a lack of brackets indicate that some interpretation is already available.
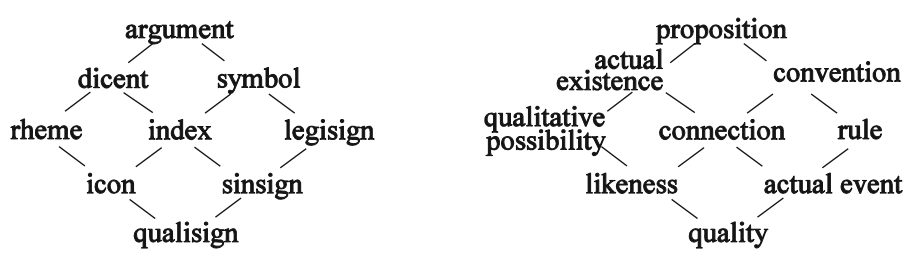

Fig. 2. Peircean sign aspects (left) and corresponding mundane terms (right)

Conforming to our goal, which is the definition of a process model of interpretation, our focus is restricted to interpretation as a sequence of events (hence in the paragraph above references to the Peircean categories are references to categorical aspects only). Following this line of thought we offer a semiotic analysis of our process model and assign sign aspects to its events [12]. See Fig.2. The goal of this slightly technical part in the next paragraph is to show that the events can be assigned to all Peircean sign aspects hence, in this sense, the process is semiotically complete.

State and effect appear as independent qualities ([q1 q2]; qualisign). Sorting of the input has the sign aspects of likeness or constituency ([q1]; icon), simultaneity ([q2]; sinsign), and connection ${ }^{2}$ ([C]; index). As a state may occur in itself, but an effect always assumes the existence of a state, qualities of the state and effect can be used for a representation of a relation of constituency and simultaneity, respectively. Abstraction of the input qualities has the sign aspects of a possible state ( 1 1; rheme), and effect (q2; legisign). By virtue of the involvement relation between state and effect, the abstract effect, which stands for the possible combinations known about the input state and effect, has the sign aspect of a habitual event. An expression of the abstract state and effect, in context ([C]), has the sign aspects of an existent or actual state $((\mathrm{q} 1, \mathrm{C})$; dicent $)$, and a conventional event $((\mathrm{q} 2, \mathrm{C}) ;$ symbol). Finally, predication, expressing a relation between the input state and effect in context has the sign aspect of a proposition which is a premise $((\mathrm{q} 1, \mathrm{C})-(\mathrm{q} 2, \mathrm{C})$; argument $)$.

\footnotetext{
${ }^{2}$ We tacitly assume that $\mathrm{C}$, representing complementary information corresponding to $\mathrm{q} 1$ and $\mathrm{q} 2$, appears together with q1 and q2 as a quality. In Fig.1 this is omitted.
} 

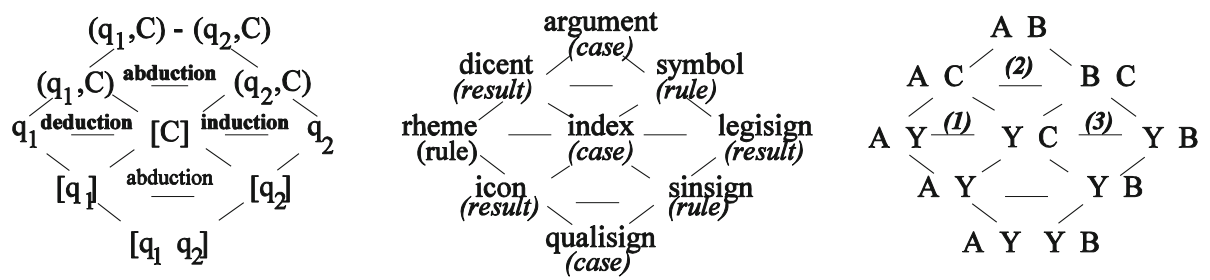

Fig. 3. A reasoning analysis of the process model. Types of reasoning aspects (left), syllogistic functions (middle), and formal expression (right). Aristotle's three figures are referred to by integers in parenthesis (see also Fig. 4). A Y and Y B designate q1 and q2 as premises

$\begin{array}{lllllll}\text { deduction } & \text { abduction } & \text { induction } & \text { figure-1 } & \text { figure-2 } & \text { figure-3 } \\ \text { rule } & \text { rule } & \text { result } & \text { Y C } & \text { B C } & \text { Y C } \\ \text { case } & \frac{\text { result }}{\text { result }} & \text { case } & \frac{\text { case }}{\text { rule }} & \frac{\text { A Y }}{\text { A C }} & \overline{\text { A B }} & \underline{\mathrm{Y} \mathrm{C}}\end{array}$

Fig. 4. A functional (left) and a formal representation of Aristotle's syllogistic figures. Deduction corresponds to figure-1, abduction to figure-2, induction to figure-3 [10].

The introduction of a semiotic analysis above was necessary in order to be able to offer a reasoning analysis of our process model. In this paper, our focus is on the relational events themselves. The reasoning aspects, syllogistic functions (following Peirce [14]), and formal expressions, associated to them are depicted in Fig. 3. In our analysis below we may refer to the events by their syllogistic terms in Fig. 4.

The generation of the final predication relation has the reasoning aspect of abduction (2). Through combining the expressions of the state (A) and effect (B), in context $(\mathrm{C})$, we raise a hypothesis concerning their relation, which is involved in a meaningful interpretation of the input interaction. The generation of a possible representation of the input state in context has the aspect of deduction (1). In this event, complementary information (Y), that the interpreting system is familiar with, is used to derive 'new' information (C) about the state (A). This is opposed to the event generating an expression of the effect in context, which has the aspect of induction (3). In this event, a habitual interpretation of the effect (B) is applied to hence tested for a new state $(\mathrm{C})$, enabled by the context $(\mathrm{Y})$. A presentation of the input qualities as cooccurring premises, by sorting, has the aspect of abduction, degenerately (in a reasoning sense).

Noting the completeness of the process model, the semiotic analysis (Fig. 2) shows the involvement of all nine Peircean sign aspects, the reasoning analysis (Fig. 3) reveals the potential of the process for a representation of all three types of reasoning as aspects of reasoning, syllogistic functions and formal expressions. By virtue of the dependency between the categories, we may assume that the above completeness (secondness) is involved in a meaningful interpretation of the input as a phenomenon as well (thirdness).

In the case of language phenomena, examples of an effect are words, sentences, paragraphs, or even entire texts, for instance, chairman statements. In this paper, we assume that in such statements, complex thoughts by the author are split into smaller fragments, in order to help the reader's comprehension. Each paragraph is meaningful 
in itself and layout is used to draw the reader's attention as well. If a paragraph is an expression of a single thought, then by virtue of the completeness of meaningful interpretation, it must represent all three types of reasoning (aspects). From this we conclude that when the line of reasoning is complex, the author of the text may wish to add formal information thereby enhancing a reasoning interpretation by the reader. We conjecture that through analyzing the form of reasoning involved in a text, a measure of plausibility and understandability by the reader can be derived.

\section{$3 \quad$ An Illustration of Corporate Disclosures}

We apply our theory to a paragraph found in a chairman's statement ${ }^{3}$ (See also Fig.5). We assume that a syntactic analysis of the input sentences is available. In our reasoning analysis below, sentences are interpreted as premises, the subject and predicate of a sentence as the minor and major terms of a premise. A common term can be found by matching. Information, such as syntactic and semantic-syntactic, necessary for this operation is assumed to be available (this may put a great burden on lexicon definition).

A sentence may function as a premise in different syllogisms. A syllogism can be interpreted from the perspective of itself, as well as from the stance of the paragraph as a whole. The import of a syllogism can be the expression of a reasoning aspect in the interpretation of the paragraph (cf. the positions in the upper 'triangle' marked by rheme, legisign and argument), but also it can be only a representation of a single sign aspect (cf. the positions in the lower 'triangle' marked by qualisign, rheme, and legisign). In the second case the involved reasoning aspect is represented degenerately, from a logical stance (see sect. 3.1 and 3.2).

S1: Rebuilding the bank is a significant challenge but I believe we now have the tools to begin turning the business around.

S2: Niall Booker, a veteran of the banking world, joined the Bank at the same time as me, and he and his new Executive Team have worked closely with the Board over the second half of last year to understand the true state of the Bank.

S3: Devising a Recapitalisation Plan acceptable to all stakeholders in very difficult circumstances, we were able to complete the Liability Management Exercise (LME) in December without which we wouldn't be here today.

S4: This could not have been accomplished without the support of the vast majority of our subordinated bondholders, customers and colleagues for which we are extremely grateful.

Fig. 5. A sample paragraph from a chairman's statement

\subsection{S1}

The first sentence, $\mathrm{S} 1$, is setting the scene (the first clause in $\mathrm{S} 1$ is setting the context). This sentence is not part of a syllogism, except as a proposition which is a conclusion.

\footnotetext{
${ }^{3}$ The Co-operative Bank plc. Annual report and account, 2013
} 
The subject ('we') and predicate ('have the tools to begin turning the business around') are brought into relation via predication. In the interpretation of the paragraph as a whole, the above two symbols are used for a definition of the icon and sinsign positions. The first clause of $\mathrm{S} 1$ is represented as complementary information in the index position ('Rebuilding', 'challenge'). See Fig. 6. Note the relation of overlapping between the abduction involved in predication (cf. S1) and in sorting (cf. paragraph).

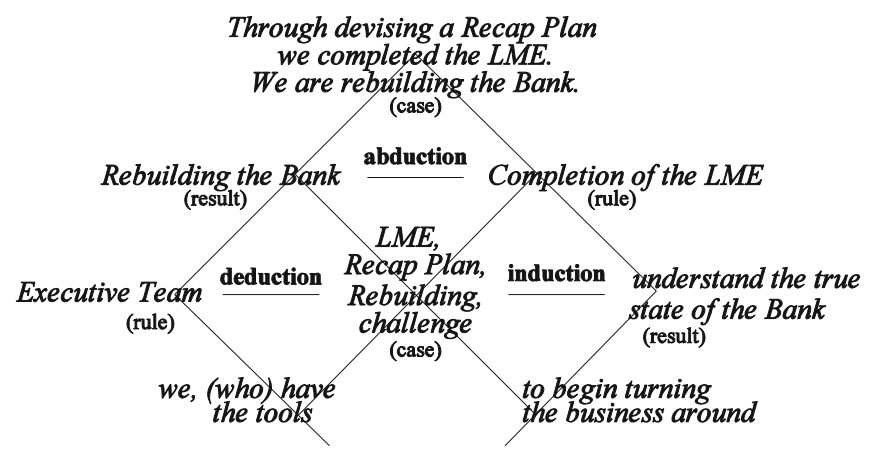

Fig. 6. A reasoning interpretation of the paragraph depicted in Fig. 5. A representation of the qualisign position is omitted. In the icon position, 'have the tools' mediates between 'we' (agent) and 'to begin turning the business around' (theme). The term, 'Recap Plan' is used as short for 'a Recapitalisation Plan acceptable to all stakeholders'. Reasoning aspects that are represented degenerately (in a logical sense) are omitted.

\section{$3.2 \mathrm{~S} 1-\mathrm{S} 2$}

$\mathrm{S} 1$ and S2 can be interpreted as premises of a syllogism exhibiting the aspects of induction (cf. Fig. 4). The common term is defined by the expressions 'Rebuilding the Bank', and by 'Niall Booker' and 'Executive Team', as theme and agent, respectively, of the predicates of S1 and S2 interpreted as major terms. The import of this syllogism in the paragraph as a whole is an expression of the legisign position: ${ }^{4}$ 'understand the true state of the Bank' (a full definition: '(they) worked closely to understand the true state of the Bank IS (enabled by) having the tools to begin turning the business around'). In addition, S1 and S2 can also be interpreted as premises of a syllogism exhibiting the aspects of deduction. This time the common term is defined by 'Rebuilding the Bank' (S1), and 'worked closely to understand the true state of the Bank' (S2). The import of this syllogism is an expression of the rheme position ${ }^{5}$ : 'Executive Team'.

\section{$3.3 \quad \mathrm{~S} 2-\mathrm{S3}$}

S2 and S3 can be interpreted as premises of a syllogism exhibiting the aspects of induction. The common term is defined by the expressions 'He and his Executive Team',

\footnotetext{
${ }^{4}$ It will be applied as result in a later syllogism exhibiting the aspects of induction.

${ }^{5}$ It will be applied as rule in a later syllogism exhibiting the aspects of deduction.
} 
and ' $w e$ '. The import of this syllogism in the analysis of the entire paragraph is a representation of the aspect of induction and an expression of the symbol position (rule): 'completion of the LME' (a full definition: '(we) were able to complete the LME IS (through) understand(ing) the true state of the Bank'). Information about 'the Liability Management Exercise', involved in the expression of the symbol position, as well as information about 'devising a Recapitalisation Plan', occurring in the first clause of S3 are represented as a context sign, in the index position ('LME', 'Recap Plan').

\section{$3.4 \quad \mathrm{~S} 1-\mathrm{S3}$}

S1 (by its first clause) and S3 can be interpreted as premises of a syllogism exhibiting the aspects of deduction. The common term is defined by 'challenge' ( $\mathrm{S} 1)$ and 'we' (S3), as theme and agent, respectively. The import of this syllogism in the paragraph as a whole is a representation of the aspect of deduction and an expression of the dicent position (result): 'Rebuilding the Bank' (a full definition: 'Rebuilding the Bank IS (was) enabled by the completion of the LME (through) devising a Recapitalisation Plan').

\section{$3.5 \quad \mathrm{S3}-\mathrm{S} 4$}

$\mathrm{S} 3$ and S4 can be interpreted as premises of a syllogism exhibiting the aspects of abduction. The common term is defined by the expressions 'complete', and 'accomplish'. The import of this syllogism in the paragraph as a whole is a representation of the aspect of abduction and an expression of the argument position (case): 'This (=the completion of the LME hence the Rebuilding of the Bank) IS (accomplished through) devising a Recapitalisation Plan' (a full definition, following paraphrasing: 'Through devising a Recapitalisation Plan we completed the LME. (This way) we are rebuilding the Bank'.

A reasoning interpretation of $\mathrm{S} 1$ and $\mathrm{S} 4$ is already involved in that of $\mathrm{S} 3$ and $\mathrm{S} 4$; a reasoning interpretation of $\mathrm{S} 2$ and $\mathrm{S} 4$ is not possible.

\section{$4 \quad$ Experimental Results}

We devised a test to measure understandability and plausibility, using two sample chairman's statements taken from 2009 and 2013 Annual Report of a UK company. Understandability was tested through questions concerning the meaning involved in the informationally not represented syllogistic aspects according to our analysis, while their meaning must be clear to the reader, by virtue of the assumed completeness of the paragraphs. Completeness offers the possibility to verify whether the syllogistic structure is a good approximation of reasoning involved in meaningful interpretation. For instance, a paragraph, which can be analyzed in inductive syllogism(s) must convey information about the reasoning aspects of an involved deduction and abduction as well that can be verified through questions. Scale questions ( $1=$ Not at all plausible, 4=Plausible) were employed to test plausibility thereby to determine the persuasiveness. The score can be used for verifying our hypothesis concerning the ordering of syllogistic schemes. We conjecture that a text mostly representing the reasoning aspects of deduction will be perceived to be more plausible than a text, which mostly representing the reasoning aspects of induction (testing) and abduction (hypotheses). A tacit goal of this 
experiment is to show that an analysis of reasoning can be analogous to syntactic parsing.

The experiment was conducted with undergraduate accounting students in a UK university. It resulted in 27 responses, from which 21 were acceptable, from a group of 70 students. These students' first language was English and can be classed as knowledgeable users of financial reports. Each student was required to read five paragraphs extracted from two chairman's statements and fill out an on-line questionnaire via Kwiksurvey, consisting of 14 multiple-choice questions. The experiment required about 20-30 minutes to complete. SPSS was used to analyze the data as part of a descriptive statistics research, which measures the occurrence of the 'correct' answers for each question. When measuring understandability, one cannot expect a single correct answer since the interpretation process among individuals is different. Therefore the answers to the type of questions were distributed in levels of 'correctness' (1=worst answer, $4=$ best answer).

We found that in questions measuring understandability, once the text is complete from a reasoning stance, $76 \%$ students were able to select the best and second best answers, while $14 \%$ chose the worst answer. In the case of incompleteness, only $9 \%$ students managed to select the best and second best answers. This indicates that completeness leads to better understandability. The results from the plausibility questions showed that the use of aspects of deductive reasoning makes the paragraphs more plausible as deductive reasoning conforms to more logical reasoning and to less persuasive reasoning. In the experiment, all questions deriving from deductive reasoning type attracted only one "not at all plausible" answer. The answers to questions involved in inductive reasoning were more dispersed than others, ranging from "plausible" to "not at all plausible". A very interesting finding was that no students chose "not at all plausible" for abductive type of reasoning questions, which is deemed to the correct answer. Instead, nearly half of the students selected "plausible" in their answers, which means that students could still be persuaded by a "good reason". This supports Fisher's (1987) narrative assumption that human beings are fundamentally storytelling creatures, therefore, the most persuasive information is not that of a logical argument, but instead a narrative rationality.

\section{$5 \quad$ Summary and Future Research}

Accounting narratives in annual reports constitute a key part of corporate disclosures. Organizations utilize narratives to communicate corporate information to various publics. Effective communication depends on the accuracy in the construction and transmission of the message between senders and readers. In this paper, we presented a practical illustration of the application of our semiotically inspired process model to interpret a text (i.e. chairman's statement), through the analysis of syllogistic forms. Our experiment results suggest that the syllogistic form analysis measure aspects of the understandability and persuasiveness. This form-oriented study contributes to the narrative studies by capturing both syntactic and semantic interpretation. The limitation of the study is that we only investigate 2 chairman statements and the scale of the experiment is small. We leave an examination of the statistical correlation between syllogistic reasoning and understandability/plausibility for further research. 


\section{References}

1. Smith, M., Jones, M.: Traditional and alternative methods of measuring the understandability of accounting narratives. Accounting, Auditing \& Accountability Journal 27, 183-208 (2014)

2. Courtis, J.K.: An Investigation into Annual Report Readability and Corporate Risk-Return Relationships. Accounting and Business Research 16, 285-294 (1986)

3. Tregidga, H., Milne, M., Kearins, K. (Re)presenting 'sustainable organizations'. Accounting, Organizations and Society 39, 477-494 (2014)

4. Aerts, W.: On the use of accounting logic as an explanatory category in narrative accounting disclosures. Accounting, Organizations and Society 19, 337-353 (1994)

5. Kothari, S.P., Xu, L., Short, J.E.: The Effect of Disclosures by Management, Analysts, and Business Press on Cost of Capital, Return Volatility, and Analyst Forecasts: A Study Using Content Analysis. Accounting Review 84, 1639-1670 (2009)

6. García Osma, B., Guillamón-Saorín, E.: Corporate governance and impression management in annual results press releases. Accounting, Organizations and Society 36, 187-208 (2011)

7. Smith, J.E., Smith, N.P.: Readability: A Measure of the Performance of the Communication Function of Financial Reporting. Accounting Review 46, 552 (1971)

8. Jones, M.J.: A Longitudinal Study of the Readability of the Chairman's Narratives in the Corporate Reports of a UK Company. Accounting and Business Research 18, 297-305 (1988)

9. Loughran, T., McDonald, B.: Measuring Readability in Financial Disclosures. Journal of Finance 69, 1643-1671 (2014)

10. Sarbo, J.J., Farkas, J.I., Van Breemen, A.J.J.: Knowledge in Formation: A Computational Theory of Interpretation. Springer, Berlin (2011)

11. Jones, M., Shoemaker, P.: Accounting narratives a review of empirical studies of content and readability. Journal of Accounting Literature 13, 142-184 (1994)

12. Sarbo, J.J.: On Well-formedness in Requirement Elicitation Processes. In: Feng, X., Liu, K., Jiang, G. (eds.) Proc. 11th Int. Conf. on Informatics and Semiotics in Organizations, Beijing, China, pp. 18-25 (2009)

13. Li, F.: The Information Content of Forward-Looking Statements in Corporate Filings-A Naive Bayesian Machine Learning Approach. Journal of Accounting Research 48, 1049-1102 (2010)

14. Peirce, C.S.: Collected papers by Charles Sanders Peirce. Harvard University Press, Cambridge (1931-1958) 